\title{
Early biomarkers of renal injury and protective effect of erythropoietin on kidneys of asphyxiated newborn rats
}

\author{
Vesna D. Stojanović, ${ }^{1,2}$, Nada M. Vučković ${ }^{1,3}$, Nenad A. Barišić ${ }^{1,2}$, Biljana Srdić ${ }^{1}$, Aleksandra D. Doronjski ${ }^{1,2}$ and \\ Amira E. Peco Antic 4,5
}

BACKGROUND: The aims of this study were to determine which of the two biomarkers of renal injury, kidney injury molecule-1 or cystatin C, is more sensitive and to evaluate whether erythropoietin protects kidneys injured by perinatal asphyxia.

METHODS: Animals were split into three groups designated as follows: AE, pups that survived perinatal asphyxia and subsequently received $2.5 \mu \mathrm{g}(0.1 \mathrm{ml})$ of darbepoetin-a (i.p.); A, the pups that survived perinatal asphyxia and received $0.1 \mathrm{ml}$ of $0.9 \% \mathrm{NaCl}$; and $\mathrm{C}$, control group. The pups were killed at different ages of life (6h, 24h, 48h, $7 \mathrm{~d}$, and $14 \mathrm{~d}$ of age; 10 rats in each subgroup). Immunohistopathological evaluation of kidneys was performed. RESULTS: At $48 \mathrm{~h}$ and on days 7 and 14, absolute injury scores were significantly lower in group AE as measured by both biomarkers. Cystatin C expression was the most intensive $6 \mathrm{~h}$ after the hypoxic event (average value of absolute injury score was 2.82) and declined over time. Expression of kidney injury molecule- 1 was less intensive, with the average value of absolute injury score being 2.02 at $6 \mathrm{~h}$ and 2.105 at $24 \mathrm{~h}$; the peak value (2.155) was recorded $48 \mathrm{~h}$ after the hypoxic event.

CONCLUSION: Erythropoietin has a protective effect on hypoxic kidneys. Cystatin $C$ is more sensitive as an early biomarker of acute kidney injury in comparison with kidney injury molecule-1.

A cute kidney injury (AKI) is often seen in neonates. Incidence of AKI among neonates in the neonatal intensive care units is $8-24 \%$. Patients with AKI have higher mortality rates and higher risk for development of chronic kidney disease (1-3). The diagnosis of AKI is traditionally based on the measurement of blood urea nitrogen and creatinine. These methods are neither sensitive nor specific for AKI. A number of recently found proteins from serum and urine have been proposed as promising markers of AKI, giving rise to the possibility of diagnosis even before blood urea nitrogen and creatinine increase. The most important among these are neutrophil gelatinase-associated lipocalin, kidney injury molecule-1 (KIM-1), cystatin C, interleukin-18, and liver-type fatty acid-binding protein (4-7).

KIM-1 is an adhesion molecule found in epithelial cells. KIM-1 and its mRNA are detectable in small amounts in the normal kidney, but, in the ischemic kidney, their concentrations increase significantly. It is believed that KIM-1 plays a role in the processes of epithelial regeneration and disposal of dead cells from the tubular lumen via phagocytosis $(8,9)$.

Cystatin $\mathrm{C}$ is a protein produced in all cells that have a nucleus. It is freely filtered in the glomerulus, completely reabsorbed in the proximal tubules, and secreted in the renal tubules. It was found that, in patients with $\mathrm{AKI}$, cystatin C levels rise $1-2 \mathrm{~d}$ earlier than do serum creatinine levels and that cystatin $\mathrm{C}$ is a better marker of glomerular filtration rate $(10,11)$.

Erythropoietin (EPO), which is produced in the adult kidney and fetal liver, is a well-known regulator of erythropoiesis in mammals. During the past decade, it has been proven that EPO receptors are expressed in other organs, such as brain, heart, and kidneys. In addition, several experimental studies have demonstrated neuroprotective, cardioprotective, and renoprotective effects of EPO after ischemia/reperfusion (I/R) injury, which are independent of its hematopoietic activity. EPO can prevent or reduce renal damage and enhance renal recovery by reducing apoptosis, by promoting neovascularization and tissue regeneration, and through its anti-inflammatory action (12-15).

The aim of our study was to determine which of the two early biomarkers of renal injury, KIM-1 or cystatin C, is a more sensitive indicator of kidney damage and whether EPO administered after birth has any protective effect on kidneys of rats damaged by perinatal asphyxia.

\section{RESULTS}

\section{Kidney Injury Molecule-1}

Distribution of the values of absolute injury scores in groups $\mathrm{AE}$ (pups that survived perinatal asphyxia and subsequently received $2.5 \mu \mathrm{g}(0.1 \mathrm{ml})$ of darbepoetin- $\alpha$ ) and A (pups that survived perinatal asphyxia and received $0.1 \mathrm{ml}$ of $0.9 \% \mathrm{NaCl}$ ) at 6 and $24 \mathrm{~h}$ after reperfusion is shown in Figure 1.

During the first $24 \mathrm{~h}$ after I/R (samples obtained at 6 and $24 \mathrm{~h}$ ), the values of absolute scores of injury were not significantly different between the groups (at $6 \mathrm{~h}: P=0.554$; and at 24 h: $P=0.161$ ).

Distribution of values of absolute injury scores in groups $\mathrm{AE}$ and $\mathrm{A}$ at $48 \mathrm{~h}$ and on days 7 and 14 after reperfusion is shown in Figure 2. 


\section{Articles | Stojanović et al.}

At $48 \mathrm{~h}$ and on days 7 and 14, the values of absolute injury scores were significantly lower in group AE (48 h: $P=0.008$; day 7: $P=0.018$; and day $14: P=0.007)$.

Immunohistochemical staining for KIM-1 in the control group was negative.

\section{Cystatin C}

Immunohistochemical staining for cystatin $\mathrm{C}$ revealed cytoplasmic granules in proximal tubular cells. In the first $24 \mathrm{~h}$ after I/R (samples obtained at 6 and $24 \mathrm{~h}$ ), the values of absolute scores of injury were not significantly different between the groups $(P>0.05)$.

At $48 \mathrm{~h}$ and on days 7 and 14, the values of the absolute scores of injury in group AE were significantly lower than the values in group $\mathrm{A}(P<0.05)$.

Distribution of values of absolute injury scores in groups AE and $A$ at $48 \mathrm{~h}$ and on days 7 and 14 after reperfusion is shown in Figure 3.

Immunohistochemical staining for cystatin $\mathrm{C}$ in the control group was negative.

\section{a}
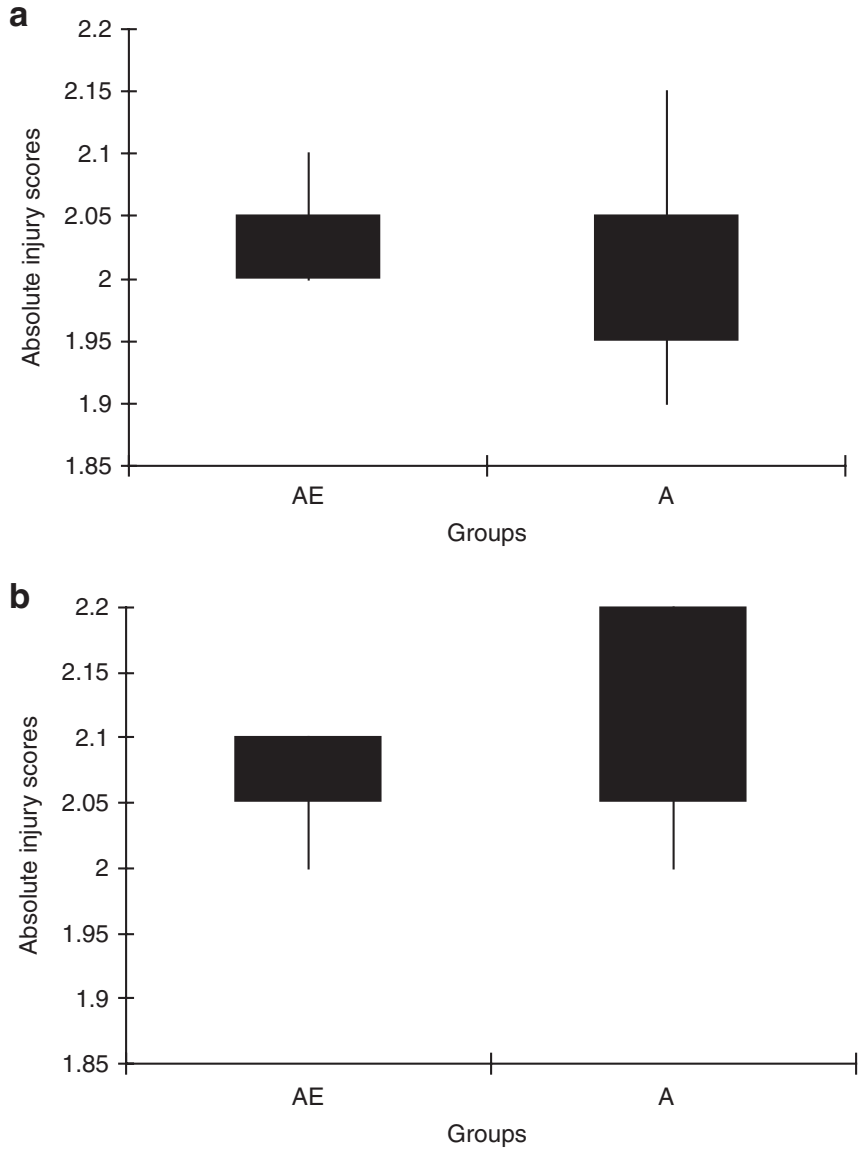

Figure 1. Distribution of values of absolute injury scores in groups $A E$ and $A$ at 6 and $24 \mathrm{~h}$ after reperfusion (immunohistochemical staining for KIM-1). (a) Distribution of values of absolute injury scores in groups $\mathrm{AE}$ and $\mathrm{A} 6 \mathrm{~h}$ after reperfusion. (b) Distribution of values of absolute injury scores in groups $\mathrm{AE}$ and $\mathrm{A} 24 \mathrm{~h}$ after reperfusion. A, pups that survived perinatal asphyxia and received $0.1 \mathrm{ml}$ of $0.9 \% \mathrm{NaCl} ; \mathrm{AE}$, pups that survived perinatal asphyxia and received $2.5 \mu \mathrm{g}(0.1 \mathrm{ml})$ of darbepoetin- $\alpha$ (i.p.); KIM-1, kidney injury molecule-1.

\section{Cystatin C vs. KIM-1}

In asphyxiated pups that received EPO, cystatin C and KIM-1 were also detectable in proximal tubular cells immediately after I/R. Cystatin C expression was the most intensive immediately after I/R (average value of absolute injury score at $6 \mathrm{~h}$
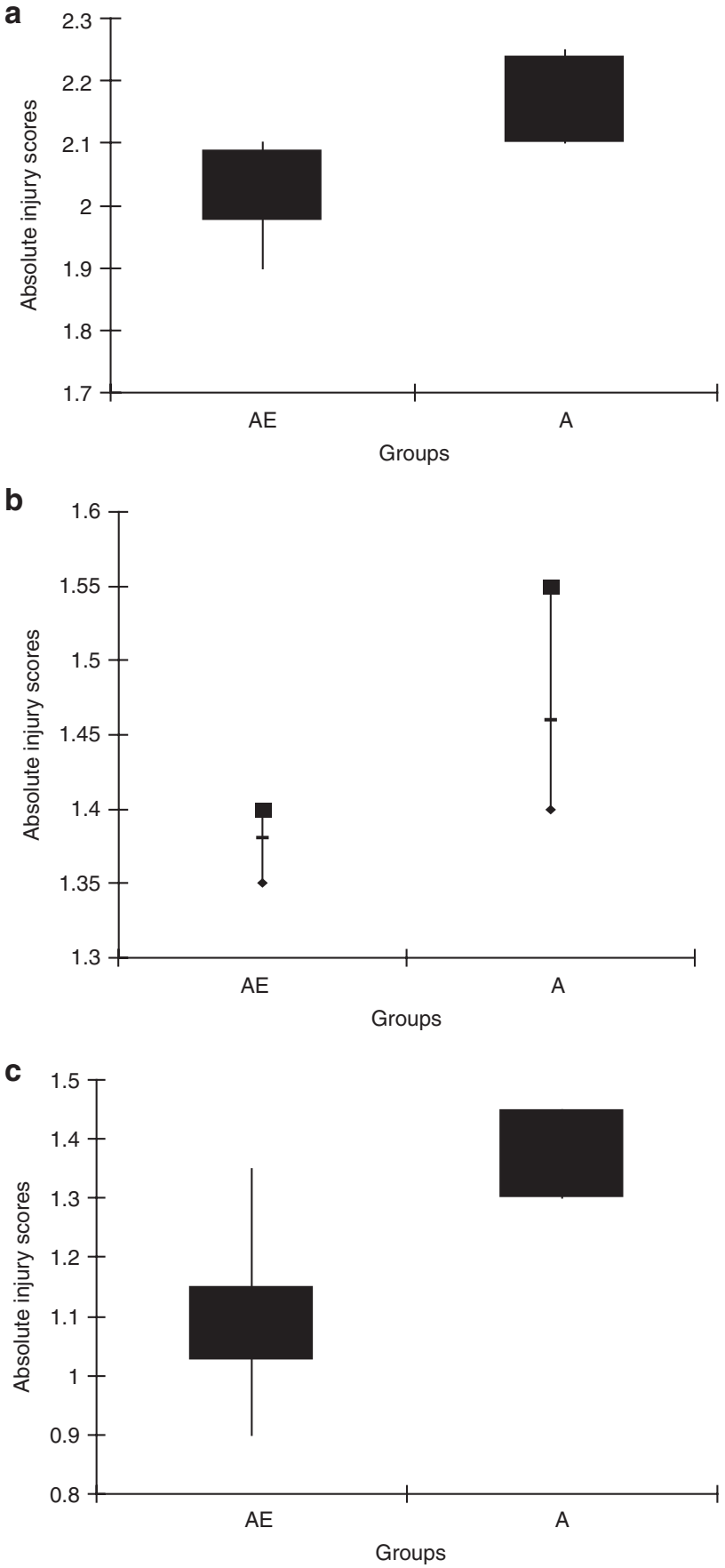

Figure 2. Distribution of values of absolute injury scores in groups $A E$ and $\mathrm{A}$ at $48 \mathrm{~h}$ and on days 7 and 14 after reperfusion (immunohistochemical staining for KIM-1). (a) Distribution of values of absolute injury scores in groups AE and $A 48 \mathrm{~h}$ after reperfusion. (b) Distribution of values of absolute injury scores in groups $A E$ and $A 7 d$ after reperfusion. (c) Distribution of values of absolute injury scores in groups $A E$ and $A 14 d$ after reperfusion. $A$, pups that survived perinatal asphyxia and received $0.1 \mathrm{ml}$ of $0.9 \% \mathrm{NaCl} ; \mathrm{AE}$, pups that survived perinatal asphyxia and received $2.5 \mu \mathrm{g}$ $(0.1 \mathrm{ml}$ ) of darbepoetin- $\alpha$ (i.p.); KIM-1, kidney injury molecule-1. 

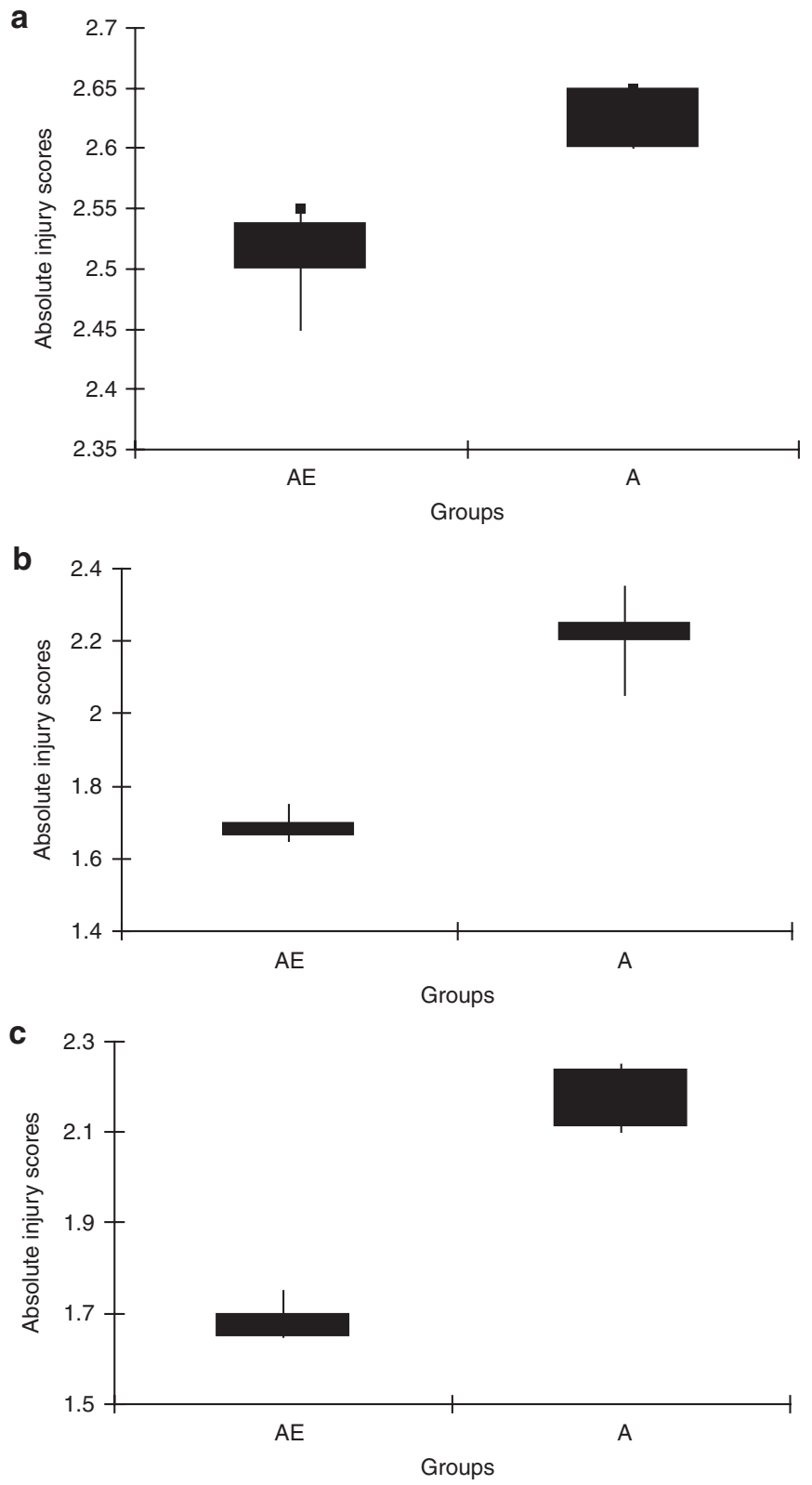

Figure 3. Distribution of values of absolute injury scores in groups $A E$ and $A$ at $48 \mathrm{~h}$ and on days 7 and 14 after reperfusion (immunohistochemical staining for cystatin C). (a) Distribution of values of absolute injury scores in groups $\mathrm{AE}$ and $\mathrm{A} 48 \mathrm{~h}$ after reperfusion. (b) Distribution of values of absolute injury scores in groups $A E$ and $A 7 \mathrm{~d}$ after reperfusion. (c) Distribution of values of absolute injury scores in groups $A E$ and $A 14 d$ after reperfusion. $A$, pups that survived perinatal asphyxia and received $0.1 \mathrm{ml}$ of $0.9 \% \mathrm{NaCl} ; \mathrm{AE}$, pups that survived perinatal asphyxia and received $2.5 \mu \mathrm{g}(0.1 \mathrm{ml})$ of darbepoetin- $\alpha$ (i.p.).

was 2.82) and declined over time (average values of absolute injury scores were $2.75,2.51,1.70$, and 1.69 at $24 \mathrm{~h}$, at $48 \mathrm{~h}$, on day 7 , and on day 14 , respectively). On the other hand, expression of KIM-1 was less intensive, and the average value of absolute injury score at $6 \mathrm{~h}$ was 2.03 . The peak value of this score was registered $24 \mathrm{~h}$ after I/R (average value of absolute injury score was 2.06), and beyond this point, the score continuously declined (48 h: 2.03; day 7: 1.38; and day 14: 1.09; Figure 4).

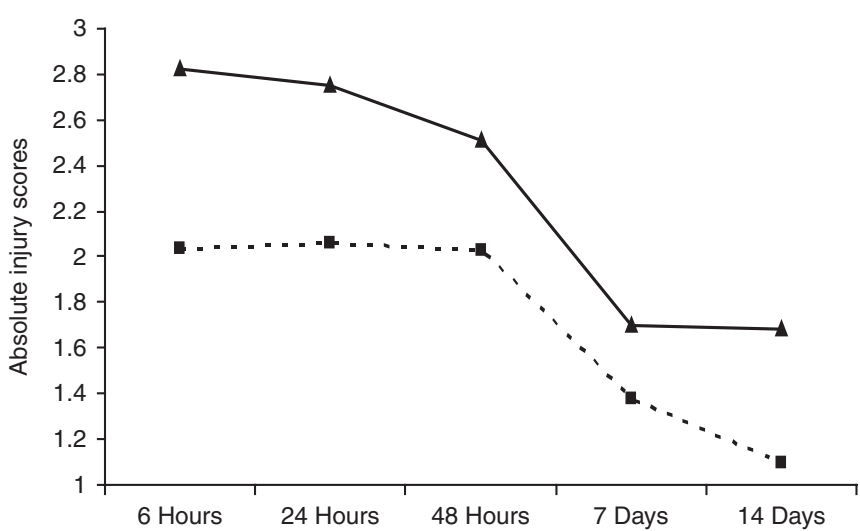

Figure 4. Trends of absolute injury scores in asphyxiated pups that received EPO (KIM-1 vs. cystatin C). Solid line: cystatin C; dashed line: KIM1. EPO, erythropoietin; KIM-1, kidney injury molecule-1.
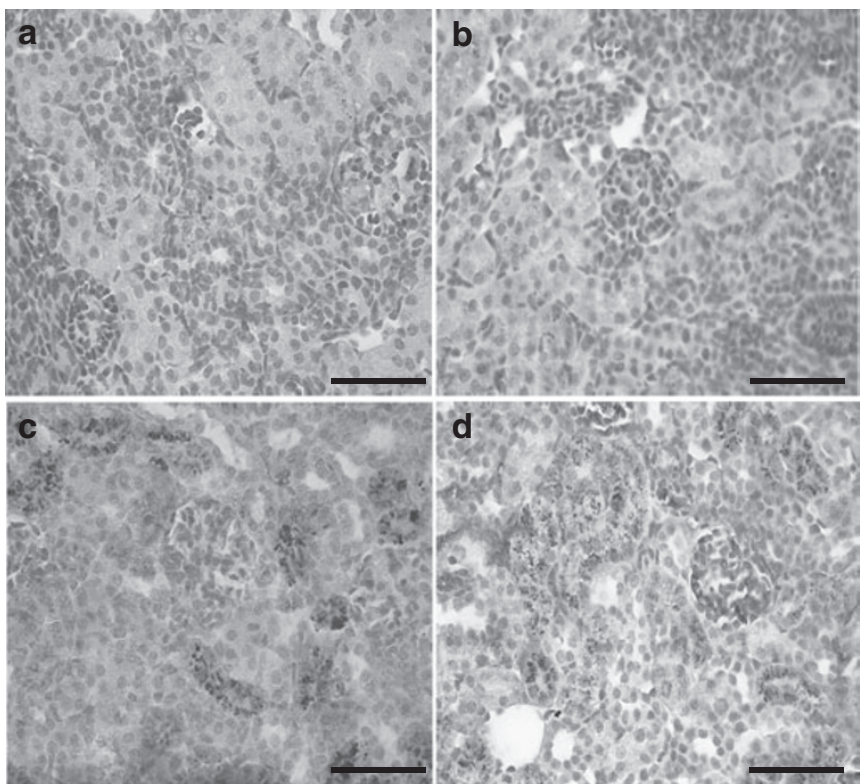

Figure 5. Immunohistochemical staining for KIM-1 and cystatin $C$ in groups $A E$ and $A$ on day 14. (a) Staining for KIM-1 in group $A E$ (magnification: $\times 400$; scale bar $=60 \mu \mathrm{m}$ ). (b) Staining for KIM-1 in group A (magnification: $\times 400$; scale bar $=60 \mu \mathrm{m}$ ). (c) Staining for cystatin $C$ in group AE (magnification: $\times 400$; scale bar $=60 \mu \mathrm{m}$ ). (d) Staining for cystatin $C$ in group $A$ (magnification: $\times 400$; scale bar $=60 \mu \mathrm{m}$ ). A, pups that survived perinatal asphyxia and received $0.1 \mathrm{ml}$ of $0.9 \% \mathrm{NaCl}$; $\mathrm{AE}$, pups that survived perinatal asphyxia and received $2.5 \mu \mathrm{g}(0.1 \mathrm{ml})$ of darbepoetin- $\alpha$ (i.p.); KIM-1, kidney injury molecule-1.

Immunohistochemical staining for KIM-1 and cystatin $\mathrm{C}$ revealed cytoplasmic granules in proximal tubular cells (Figure 5).

\section{DISCUSSION}

Creatinine levels begin to rise when $50 \%$ of renal function is already lost. Changes in serum creatinine and blood urea nitrogen primarily reflect functional changes in filtration capacity and are not actual "markers of damage." The increase in serum creatinine levels occurs $48-72 \mathrm{~h}$ after the initial renal insult. Moreover, in advanced renal disease, there may be just 


\section{Articles | Stojanovićet al.}

minimal or no changes in the serum creatinine levels because of renal reserve or enhanced tubular secretion of creatinine. Identification of early biomarkers of tubular damage would be useful for early diagnosis and therapy and would aid in the evaluation of the effects of treatment. Introduction of early treatment may significantly reduce mortality associated with AKI $(10,16,17)$.

Early biomarkers of kidney injury may have multiple applications in patients with AKI: differentiation of AKI subtypes (prerenal, intrinsic, or postrenal), determination of etiology (ischemia, toxins, sepsis, or a combination), differentiation of AKI from other forms of acute kidney disease (infection, glomerulonephritis, or interstitial nephritis), prediction of severity of AKI, and evaluation of the course of the disease and response to therapy $(10,18,19)$.

The most studied early biomarkers of kidney injury (in addition to neutrophil gelatinase-associated lipocalin and interleukin-18) are cystatin C and KIM-1, which were used in our study.

A number of studies have found that cystatin C is a very good indicator and a useful tool for the early diagnosis of AKI. In pediatric patients admitted to the intensive care unit, cystatin C enables diagnosis of AKI up to $24-48 \mathrm{~h}$ before clinical diagnosis $(20,21)$. Askenazi et al. (22) found that the levels of urinary cystatin $\mathrm{C}$ were significantly higher in patients with AKI compared with the levels in those without AKI. In addition, the same authors found that the levels of urinary KIM-1 were higher in patients with AKI; however, that difference was not statistically significant.

On the other hand, Royakkers et al. (23) found that serum and urinary cystatin $\mathrm{C}$ is a poor biomarker for AKI. However, Krawczeski et al. (20) found that serum cystatin C is a good early predictor of AKI and a good indicator of clinical outcome in pediatric patients after cardiopulmonary bypass. In these studies, the diagnosis of AKI was predicted with great certainty within $12 \mathrm{~h}$ of surgery. Similarly, Nguyen et al. (10) found that the values of serum cystatin C levels significantly rise within $12 \mathrm{~h}$ after heart surgery.

Elmas et al. (24) found that serum cystatin C is an independent predictor of AKI in premature newborns with respiratory distress syndrome. Kandasamy et al. (25) analyzed 10 studies that assessed the sensitivity of cystatin $\mathrm{C}$ as an early biomarker of AKI in neonates and found that it can be successfully used for evaluation of renal function in sick neonates (sepsis-induced AKI and congenital renal anomalies). Their conclusion was that the number of studies that address this issue is small and that there are no studies linking cystatin C levels with short- and long-term outcomes in sick neonates.

Over the past decade, some authors have suggested that KIM-1 is a marker of injury of proximal tubules in proteinuric, toxic, and ischemic renal disease. Recently, great attention has been paid to the physiologic role of KIM-1 in modulation of tubular injury and recovery.

Urinary KIM-1 is not a sensitive indicator of progression of AKI in the first $6 \mathrm{~h}$ after $\mathrm{I} / \mathrm{R}$. The function and real role of KIM-1 are still unknown. KIM-1 may be very important for evaluation of patients with already-established diagnosis of AKI. Extensive expression of KIM-1 is detected in proximal tubular cells of patients with biopsy-proven acute tubular necrosis. A unit increase in normalized KIM-1 level was associated with a $>12$-fold risk for acute tubular necrosis (26).

KIM-1 is known as T-cell immunoglobulin mucin-like domain 1 and is expressed on the surface of $T$ cells. After $I / R$, KIM-1 expression persists during the first phase of recovery, indicating that KIM-1 is not always associated with a deleterious outcome. Moreover, if renal damage is reduced with renoprotective therapy, KIM- 1 expression is also reduced. Han et al. (27) found that KIM-1 is a more specific marker of ischemic tubular damage in comparison with other molecules that were previously investigated. They showed that KIM-1 may be detected in urine within $12 \mathrm{~h}$ after the initial ischemic renal insult. This occurs before the start of regeneration of the epithelium and persists over time. In animal models, it is seen that KIM-1 is present coincident with the appearance of markers of epithelial cell dedifferentiation and proliferation (27).

Using an immunofluorescence technique, Humphreys et al. (28) found strong apical KIM-1 staining in injured kidneys. In our experiment, however, KIM-1 immunohistochemistry (as seen with light microscopy) revealed cytoplasmic granules in the epithelial cells of proximal tubules. Moreover, in our material, granular staining was seen in various parts of the cells' cytoplasm. We think that these different findings were a consequence of different techniques of analysis; we were not able to precisely locate stained areas within the specific parts of cells.

The question is whether the tubular KIM-1 expression is involved in the process of recovery (repair) and/or damage or whether it is merely the result of ischemia, proteinuria, or renal fibrosis (29).

Nguyen et al. (10) reported that KIM-1 levels increase by $40 \%$ in the first $2 \mathrm{~h}$ after surgery and by $>100 \%$ after $24 \mathrm{~h}$.

Han et al. (27) indicate that a "panel of urinary biomarkers" can predict tubular damage better than a single urinary biomarker.

Our study has shown that cystatin C is a more sensitive early biomarker of renal injury compared with KIM-1. Both biomarkers were extensively expressed in the proximal renal tubules immediately after the hypoxic event, but staining with cystatin $\mathrm{C}$ was more intensive.

The maximal expression of cystatin $\mathrm{C}$ was registered as soon as $6 \mathrm{~h}$ after I/R. The peak of KIM-1 expression was detected $48 \mathrm{~h}$ after I/R. In asphyxiated pups, expression of both biomarkers stabilized after $7 \mathrm{~d}$ and showed no further decline until day 14. In pups that received EPO immediately after exposure to hypoxia, the expression of cystatin $\mathrm{C}$ was unchanged after $7 \mathrm{~d}$. On the other hand, expression of KIM-1 continued to decline. In our study, we tracked changes in expression of the two biomarkers during a 2-wk period after the initial insult. The faster decline of KIM-1 is probably enforced by renoprotective agents, such as, in our case, EPO. de Borst et al. (8) found that application of renoprotective agents that ameliorate renal injury leads to decreased expression of KIM-1 in rodents, during a period of 7-11 wk of follow-up. This supports the 
hypothesis that tubular injury is reversible. Due to the limited follow-up period, we do not have enough data that may give the final answer regarding whether tubular injury may be completely ameliorated.

Preventive application of recombinant human EPO mimics ischemic preconditioning and protects neural, cardiac, and renal cells of various stressors such as ischemia or cytotoxic drugs. On the other hand, recombinant human EPO has shown beneficial effects even when given after stressogenous events (e.g., after cardiac, renal, or cerebral ischemia), which proves that it can be used not only for prevention but also for the treatment of ischemia-induced injuries (12).

In addition to liver and kidneys, EPO is secreted by peripheral endothelial cells, vascular smooth muscle cells, neurons, astrocytes, microglia, and cardiomyocytes. Moreover, these cells have EPO receptors (30).

Several recent studies have shown that EPO protects kidneys from damage and improves renal function after $I / R$ injury. EPO may prevent or reduce renal damage and enhance renal recovery by reducing apoptosis, by promoting neovascularization and tissue regeneration, and through its anti-inflammatory action (31-34).

Our study confirmed the protective effect of EPO on hypoxic kidney injury. On days 7 and 14 after I/R, in rats that received EPO, expression of biomarkers of renal injury was significantly lower than the expression level in rats that did not receive any treatment.

\section{Conclusion}

Cystatin C is a more sensitive early biomarker of AKI in comparison with KIM-1. EPO has favorable effects on hypoxic kidneys.

\section{METHODS}

Adult Wistar rats and pups were used for the experiment. The animals were maintained in controlled environmental conditions, with food and water provided ad libitum. The experiment was approved by the Ethical Committee on Animal Care and Use of the University of Novi Sad.

The phase of sexual cycle of the females was determined by observing serial vaginal smears by light microscopy. Females in the proestrus phase were mated with adult males. Date of conception was determined by serial observation of vaginal smears (by confirmation of sperm in the smears and the absence of further sexual cycling). On day 22 of gestation, pregnant females were subjected to general anesthesia (ketamine, $90 \mathrm{mg} / \mathrm{kg}$ ), and laparotomy was performed. Blood vessels of the uterus were ligated, and the uterus was removed and then submersed in a bath tub with $0.9 \% \mathrm{NaCl}$, heated at $38^{\circ} \mathrm{C}$, and retained in those conditions for $14 \mathrm{~min}$. Pups were delivered by cesarean section. The interval between the first and the last pup removed from the womb was $<1 \mathrm{~min}$. Thus, the total time of asphyxia was 15 min. Immediately after birth, the pups were reanimated (sweep, aspiration, and tactile stimulation).

The pups were randomly assigned to one of the following three groups: (i) group AE comprised pups that survived perinatal asphyxia and subsequently received $2.5 \mu \mathrm{g}(0.1 \mathrm{ml})$ of darbepoetin- $\alpha$ (Aranesp; Amgen, Thousand Oaks, CA) i.p. (total of 50 pups); (ii) group A, which contained pups that survived perinatal asphyxia and received $0.1 \mathrm{ml} 0.9 \% \mathrm{NaCl}$ (i.p.) (total of 50 pups); and (iii) group C (control), which comprised pups that were delivered by cesarean section without asphyxia and that received $0.1 \mathrm{ml} 0.9 \% \mathrm{NaCl}$ (i.p.) (total of 50 pups).
The pups were adequately labeled and handed to surrogate mothers. The pups were killed at different ages of life $(6 \mathrm{~h}, 24 \mathrm{~h}, 48 \mathrm{~h}, 7 \mathrm{~d}$, and $14 \mathrm{~d}$ of age -10 rats in each subgroup). After killing, both kidneys were removed and embedded in paraffin. All samples were stained with hematoxylin and eosin, periodic acid-Schiff stain, and Mallory's combination stain. Subsequently, immunohistochemical staining was performed. Tissue samples were treated with antibody to cystatin C (Rat Cystatin C Affinity Purified Polyclonal Ab and Sheep IgG; R\&D Systems, Minneapolis, MN) and anti-rat T-cell immunoglobulin mucin-like domain 1 antibody (anti-rat T-cell immunoglobulin mucin-like domain $1 /$ kidney injury molecule 1 /hepatitis A virus cellular receptor 1 antibody, R\&D Systems). Each sample was labeled with a unique code and was examined by a pathologist. The pathologist assessed the degree of damage and scored it. Tissue slides were examined two times, and the marks were compared. If they were not the same, then a third analysis was performed.

Immunohistochemistry revealed cytoplasmic granules in the epithelial cells of proximal tubules. On each slide, the quality of staining was confirmed using positive and negative controls (surrounding adipose tissue and urethral epithelium).

Grading (evaluation of the intensity of injury) was semiquantitative. We defined four levels of intensity of renal injury and marked them with grades $(0,1,2$, and 3$)$. The intensity of injury was measured on the basis of the estimated number of granules in the cytoplasm, the intensity of the color, and the sizes of the granules. Larger granules, intensively colored, and more numerous in number were scored as grade 3; medium-sized ones and in smaller number were denoted as 2 ; presence of few, small granules was scored as 1 , and the absence of granules was scored with 0 .

The assessment of the extent of injury was based on the number of cells containing colored granules (e.g., if all cells in the proximal tubules contain granules, then the extent of injury is $100 \%$; if half of the cells of the proximal tubules have granules in the cytoplasm, then the extent of injury is $50 \%$, and so on).

On the basis of the intensity (grades) and extent of injury (percentages), we calculated the absolute score of injury for each tissue sample. Absolute score of injury was calculated as the sum of the products of the intensity (grades) and extent of injury (percentages) for each specimen (e.g., if there were $70 \%$ of cells with grade 3 and $30 \%$ of cells with grade 2 in a sample, the absolute score of injury for that specimen was $0.70 \times 3+0.30 \times 2=2.70$ ).

Statistical analysis was performed using the commercial software "SOFA Statistics" data analysis software system (version 1.3.3; Released with open source AGPL3 license (C) 2009-14 Paton-Simpson \& Associates Ltd). Significance was tested using probability limits of 95\% $(P<0.05)$.

\section{STATEMENT OF FINANCIAL SUPPORT}

The study was performed as part of the project "The role of biomarkers in early detection of renal impairment and monitoring of therapeutic response in children," supported by the Ministry of Education, Science and Technological Development of the Republic of Serbia, by grant ON175079-3.

Disclosure: The authors have nothing else to disclose.

\section{REFERENCES}

1. Askenazi DJ, Namasiwayam A, Goldstein SL. Acute kidney injury in critically ill newborns: What do we know? What do we need to learn? Ped Nephrol 2009;24:265-274.

2. Gupta BD, Sharma P, Bagla J, Parakh M, Soni JP. Renal failure in asphyxiated neonates. Indian Pediatr 2005;42:928-34.

3. Agras PI, Tarcan A, Baskin E, Cengiz N, Gürakan B, Saatci U. Acute renal failure in the neonatal period. Ren Fail 2004;26:305-9.

4. Trof RJ, Di Maggio F, Leemreis J, Groeneveld AB. Biomarkers of acute renal injury and renal failure. Shock 2006;26:245-53.

5. Liangos O, Perianayagam MC, Vaidya VS, et al. Urinary N-acetyl-beta(D)-glucosaminidase activity and kidney injury molecule-1 level are associated with adverse outcomes in acute renal failure. J Am Soc Nephrol 2007;18:904-12. 
6. Finney H, Newman DJ, Thakkar H, Fell JM, Price CP. Reference ranges for plasma cystatin $\mathrm{C}$ and creatinine measurements in premature infants, neonates, and older children. Arch Dis Child 2000;82:71-5.

7. Coca SG, Yalavarthy R, Concato J, Parikh CR. Biomarkers for the diagnosis and risk stratification of acute kidney injury: a systematic review. Kidney Int 2008;73:1008-16.

8. de Borst MH, van Timmeren MM, Vaidya VS, et al. Induction of kidney injury molecule-1 in homozygous Ren2 rats is attenuated by blockade of the renin-angiotensin system or p38 MAP kinase. Am J Physiol Renal Physiol 2007;292:F313-20.

9. Han WK, Bailly V, Abichandani R, Thadhani R, Bonventre JV. Kidney injury molecule-1 (KIM-1): a novel biomarker for human renal proximal tubule injury. Kidney Int 2002;62:237-44.

10. Nguyen MT, Devarajan P. Biomarkers for the early detection of acute kidney injury. Pediatr Nephrol 2008;23:2151-7.

11. Herget-Rosenthal S, Marggraf G, Hüsing J, et al. Early detection of acute renal failure by serum cystatin C. Kidney Int 2004;66:1115-22.

12. Joyeux-Faure M. Cellular protection by erythropoietin: new therapeutic implications? J Pharmacol Exp Ther 2007;323:759-62.

13. Bogoyevitch MA. An update on the cardiac effects of erythropoietin cardioprotection by erythropoietin and the lessons learnt from studies in neuroprotection. Cardiovasc Res 2004;63:208-16.

14. Chong ZZ, Kang JQ, Maiese K. Hematopoietic factor erythropoietin fosters neuroprotection through novel signal transduction cascades. J Cereb Blood Flow Metab 2002;22:503-14.

15. Moore E, Bellomo R. Erythropoietin (EPO) in acute kidney injury. Ann Intensive Care 2011;1:3.

16. Mak RH. Acute kidney injury in children: the dawn of a new era. Pediatr Nephrol 2008;23:2147-9.

17. Goldstein SL. Pediatric acute kidney injury: it's time for real progress. Pediatr Nephrol 2006;21:891-5.

18. American Society of Nephrology. American Society of Nephrology Renal Research Report. J Am Soc Nephrol 2005;16:1886-1893.

19. Zerhouni E. The NIH roadmap. Science 2003;302:63-65.

20. Krawczeski CD, Vandevoorde RG, Kathman T, et al. Serum cystatin C is an early predictive biomarker of acute kidney injury after pediatric cardiopulmonary bypass. Clin J Am Soc Nephrol 2010;5:1552-7.
21. Zaffanello M, Franchini M, Fanos V. Is serum cystatin-C a suitable marker of renal function in children? Ann Clin Lab Sci 2007;37:233-40.

22. Askenazi DJ, Koralkar R, Hundley HE, et al. Urine biomarkers predict acute kidney injury in newborns. J Pediatr 2012;161:270-5.e1.

23. Royakkers AA, Korevaar JC, van Suijlen JD, et al. Serum and urine cystatin $\mathrm{C}$ are poor biomarkers for acute kidney injury and renal replacement therapy. Intensive Care Med 2011;37:493-501.

24. Elmas AT, Tabel Y, Elmas ON. Serum cystatin C predicts acute kidney injury in preterm neonates with respiratory distress syndrome. Pediatr Nephrol 2013;28:477-84.

25. Kandasamy Y, Smith R, Wright IM. Measuring cystatin C to determine renal function in neonates. Pediatr Crit Care Med 2013;14:318-22.

26. Vaidya VS, Remirez V, Ichimura T, Bobadilla NA, Bonventre JV. Urinary kidney injury molecule-1: a sensitive quantitative biomarker for early detection of kidney tubular injury. Am J Physiol Renal Physiol 2006;290:F519-F529.

27. Han WK, Wagener G, Zhu Y, Wang S, Lee HT. Urinary biomarkers in the early detection of acute kidney injury after cardiac surgery. Clin J Am Soc Nephrol 2009;4:873-82.

28. Humphreys BD, Czerniak S, DiRocco DP, Hasnain W, Cheema R, Bonventre JV. Repair of injured proximal tubule does not involve specialized progenitors. Proc Natl Acad Sci USA 2011;108:9226-31.

29. Bonventre JV. Kidney injury molecule-1 (KIM-1): a urinary biomarker and much more. Nephrol Dial Transplant 2009;24:3265-8.

30. Maiese K, Li F, Chong ZZ. Erythropoietin in the brain: can the promise to protect be fulfilled? Trends Pharmacol Sci 2004;25:577-83.

31. Ates E, Yalcin AU, Yilmaz S, Koken T, Tokyol C, Ates E. Protective effect of erythropoietin on renal ischemia and repercussion injury. ANZ J Surg 2005;75:1100-1105.

32. Sharples EJ, Patel N, Brown P, et al. Erythropoietin protects the kidney against the injury and dysfunction caused by ischemia-reperfusion. J Am Soc Nephrol 2004;15:2115-24.

33. Spandou E, Tsouchnikas I, Karkavelas G, Dounousi E, Simenidou C, Guiba-Tziampiri O. Erythropoietin attenuates renal injury in experimental acute renal failure ischaemic/reperfusion model. Nephrol Dial Transplant 2006;21:330-336.

34. Vesey DA, Cheung C, Pat B, Endre Z, Gobé G, Johnson DW. Erythropoietin protects against ischaemic acute renal injury. Nephrol Dial Transplant 2004;19:348-55. 